\title{
NOUVELLE
}

\section{La mitophagie : une stratégie du virus d'Epstein-Barr pour échapper à l'immunité innée}

Damien Glon, Marion Lussignol, Audrey Esclatine
Université Paris-Saclay, CEA, CNRS, Institut de biologie intégrative de la cellule (I2BC), 5 rue Jean-Baptiste Clément, 91198 Gif-sur-yvette, France.

audrey.esclatine@universite-paris-saclay.fr
> Les virus ont développé différentes stratégies pour échapper à l'immunité cellulaire afin d'assurer leur survie au cours de l'évolution. Les virus de la famille des Herpesviridae en sont un bon exemple: ces virus persistent à vie dans l'organisme hôte grâce à leur faculté à entrer dans une phase de latence dans les cellules qu'ils infectent. Sous l'effet de divers stress, ces virus peuvent ensuite se réactiver et reprendre un cycle productif. Le virus d'Epstein-Barr (EBV) est un $\gamma$-herpesvirinae ubiquitaire, car on estime qu'environ $90 \%$ de la population mondiale a développé des anticorps contre ce virus. L'EBV infecte principalement les cellules épithéliales de l'oropharynx ainsi que les lymphocytes B. II est l'agent causal de la mononucléose infectieuse, et peut également être responsable de certains cancers, comme le lymphome de Burkitt ou le carcinome du nasopharynx.

La protéine BHRFl (BamHI right forward 1), codée par l'EBV, est un homologue de $\mathrm{Bcl}-2$, une protéine impliquée dans divers processus cellulaires, comme l'apoptose ou l'autophagie [1]. La première fonction connue de BHRFl est sa capacité à bloquer l'apoptose, en relation avec sa localisation mitochondriale [2], mais nous avons récemment montré, avec nos collaborateurs, que BHRFl perturbe aussi la dynamique et le fonctionnement des mitochondries [3]. Les mitochondries sont des organites dynamiques qui subissent des processus de fission et de fusion afin de former un réseau et de maintenir leur homéostasie [4]. Lorsque le réseau mitochondrial est altéré, certaines fonctions mitochondriales le sont également. Les mitochondries jouent un rôle important lors de l'induction de l'immunité innée antivirale [5]. La protéine virale BHRFl, en agissant sur les mitochondries, est capable d'inhiber cette immunité [3].

\section{BHRF1 perturbe l'organisation}

des mitochondries pour former des mito-agrésomes

Lorsque la protéine BHRFl est exprimée dans des cellules épithéliales, elle entraîne d'importantes modifications de la longueur des mitochondries et de leur organisation dans la cellule. $\varepsilon n$ effet, BHRFl induit la fragmentation des mitochondries par un processus de fission impliquant, entre autres, la protéine Drpl (dynamin-related protein 1) [6]. BHRFl induit également l'agrégation des mitochondries à proximité du noyau, sous la forme de structures appelées mito-agrésomes (Figure 1). Cette agrégation des mitochondries est aussi observée en contexte infectieux, lorsque le cycle productif de l'EBV est réactivé dans des cellules épithéliales ou des lymphocytes B infectés par le virus de manière latente.

\section{Les mito-agrésomes permettent} l'induction de la mitophagie L'autophagie est un mécanisme, conservé chez tous les organismes eucaryotes, qui permet la dégradation de constituants cellulaires, tels que des protéines de longue durée de vie et des organites. Elle se déroule en plusieurs 


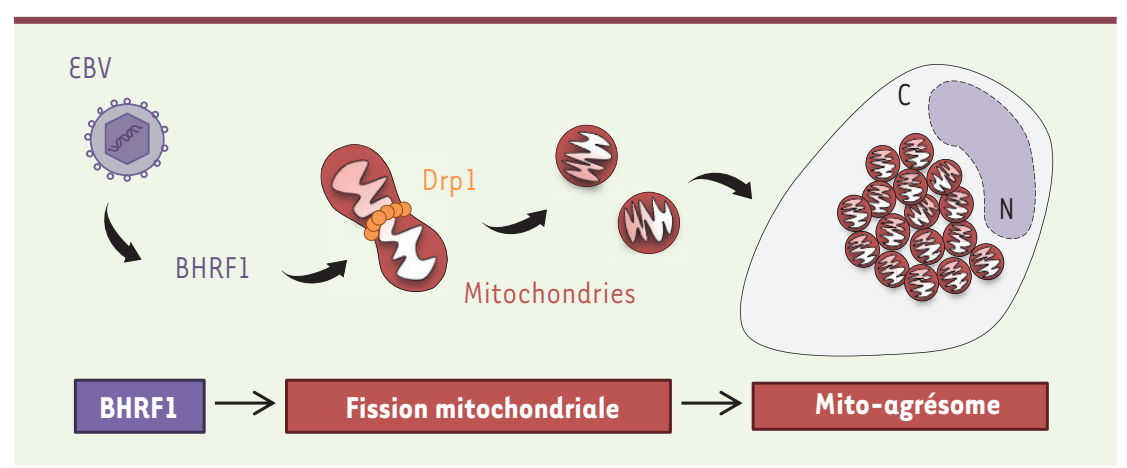

étapes, et implique des vésicules à double membrane (autophagosomes) capables d'englober des portions de cytoplasme, qui seront dégradées à la suite de la fusion de l'autophagosome avec le lysosome $(\rightarrow)$.

$(\rightarrow)$ Voir le numéro thématique Autophagie, $\mathrm{m} / \mathrm{s} \mathrm{n}^{\circ} 3$, mars 2017 , pages 213-340

Des protéines de

la famille $\mathrm{Bcl}-2$ sont connues pour moduler l'autophagie. La plupart I'inhibent, comme $\mathrm{Bcl}-2$ ou Bcl- $X_{L}$, en interagissant avec le régulateur bécline 1 , tandis que d'autres l'activent, comme BAD, Bim ou PUMA [1]. BHRFl, qui possède deux domaines d'homologie à $\mathrm{Bcl}-2$, stimule l'autophagie, entraînant une importante accumulation de vésicules autophagiques [3]. Cette induction de l'auto-
Figure 1. BHRF1 induit la formation de mitoagrésomes. BHRFl, une protéine codée par l'EBV, se localise au niveau des mitochondries pour en modifier l'organisation. Cette protéine virale induit tout d'abord la fragmentation des mitochondries en recrutant et activant Drpl. Elle entraîne ensuite l'agrégation des mitochondries à proximité du noyau, formant ainsi des mito-agrésomes. $\mathrm{N}$ : noyau ; C: cytoplasme.

phagie est nécessaire pour la formation des mito-agrésomes.

L'homéostasie mitochondriale implique le processus autophagique, notamment pour dégrader des mitochondries surnuméraires ou endommagées [7]. Cette dégradation sélective des mitochondries est appelée mitophagie [8]. Certaines études ont montré que la fission mitochondriale ainsi que la formation de

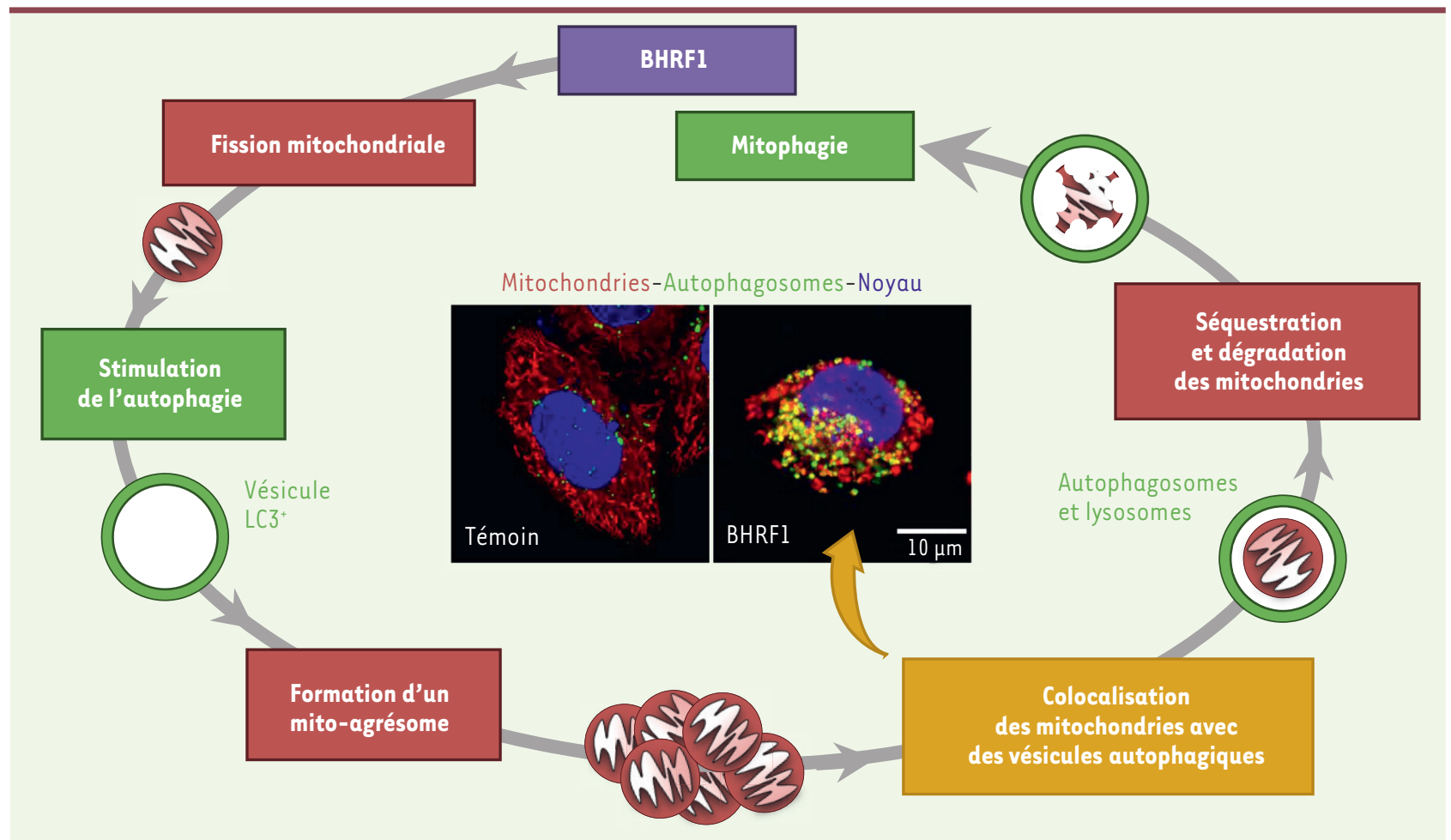

Figure 2. BHRFI induit la mitophagie. À la suite de la fission mitochondriale, BHRFl stimule l'autophagie en accumulant des autophagosomes (vésicules $L C 3^{+}$), ce qui est nécessaire à la formation des mito-agrésomes. Dans ces mito-agrésomes, une partie des mitochondries est contenue dans des vésicules qui ont un $\mathrm{pH}$ acide. En microscopie confocale, on observe que certaines mitochondries sont colocalisées avec des autophagosomes (marquage jaune sur la photo; échelle $=10 \mu \mathrm{m}$ ) et des lysosomes. Les mitochondries sont donc contenues dans des vésicules autophagiques. De plus, cette séquestration des mitochondries est suivie de la dégradation d'une partie d'entre elles. 
mito-agrésomes facilitaient l'induction de la mitophagie $[9,10]$. Compte tenu de l'impact de BHRFl sur les mitochondries et l'autophagie, une potentielle activation de la mitophagie par BHRFl a été explorée. Grâce à des sondes fluorescentes sensibles au $\mathrm{pH}$, on peut observer qu'une partie des mitochondries, sous forme de mito-agrésomes induits par BHRFl, est présente dans des compartiments qui ont un $\mathrm{pH}$ acide et identifiés comme étant des vésicules autophagiques (autophagosomes et lysosomes). Certaines de ces mitochondries sont dégradées, confirmant l'induction de la mitophagie par BHRFl (Figure 2).

\section{Inhibition de la réponse antivirale par BHRF1}

Les mitochondries possèdent à leur surface des récepteurs MAVS (mitochondrial antiviral signaling) qui sont indirectement impliqués dans la détection des infections virales. Ainsi, ces récepteurs MAVS peuvent être activés à la suite de la détection par l'hélicase RIG-I (retinoic acid-inducible gene I) de la présence d'ARN viraux dans le cytoplasme de la cellule. Après son activation, MAVS induit une cascade de signalisation qui aboutit à la synthèse d'interférons (IFN), cytokines aux propriétés antivirales [11]. Ainsi, les mitochondries sont des organites pivots dans l'établissement de la réponse immunitaire innée.

La séquestration des mitochondries dans des vésicules autophagiques et leur dégradation sont autant d'éléments suggérant que BHRFl pouvait altérer la réponse cellulaire induite par l'EBV. Cette hypothèse a été testée, notamment en mesurant le niveau de la réponse IFN grâce à un système rapporteur permettant d'évaluer l'activation du promoteur du gène codant I'IFN- $\beta$. Nous avons ainsi pu montrer que BHRFl bloque l'initiation de la réponse IFN. De plus, cet effet inhibiteur dépend à la fois de l'autophagie et de la fission mitochondriale (Figure 3 ).

La protéine BHRFl joue donc un rôle important dans l'infection par l'EBV en lui permettant d'échapper aux défenses immunitaires innées de la cellule infectée. Cette découverte étaye I'hypothèse selon laquelle BHRFl pourrait participer à la tumorigenèse associée aux infections par EBV. En effet, l'expression de BHRFl au cours de la période de latence a été associée à une plus grande résistance des cellules aux traitements anti-tumoraux [12]. Compte tenu des propriétés antiprolifératives des IFN de type I et II [13], BHRFl, en inhibant la production de I'IFN- $\beta$ (appartenant aux IFN de type I) pourrait favoriser le développement de cancers.

\section{Détruire pour survivre : une stratégie} commune à différents virus

Plusieurs autres études ont révélé comment différents virus modulent la dynamique et le devenir des mitochondries afin de contrecarrer l'immunité innée. Le virus de l'hépatite $C$, notamment, induit d'importantes modifications mitochondriales dans les cellules qu'il infecte afin de réduire la défense antivirale et améliorer sa réplication [14]. La protéine non structurale $\mathrm{PBl}-\mathrm{F} 2$ du virus de la grippe $A$ induit la dégradation de MAVS par mitophagie pour atténuer l'immunité innée [15]. L'ensemble de ces découvertes suggère que la dégradation des mitochondries induite à la suite d'une infection virale constituerait un mécanisme proviral, car elle permettrait d'atténuer les défenses innées de la cellule et d'assurer la persistance virale. $\diamond$

Mitophagy: a strategy

of the Epstein-Barr virus to evade innate immunity

\section{REMERCIEMENTS}

Ces travaux ont été réalisés grâce à des financements provenant du CNRS, de l'université ParisSaclay, du DIM MALINF Région ille-de-France, et de l'agence nationale de la recherche (ANR14-(E14-0022).

\section{LIENS D'INTÉRÊT}

Les auteurs déclarent n'avoir aucun lien d'intérêt concernant les données publiées dans cet article.

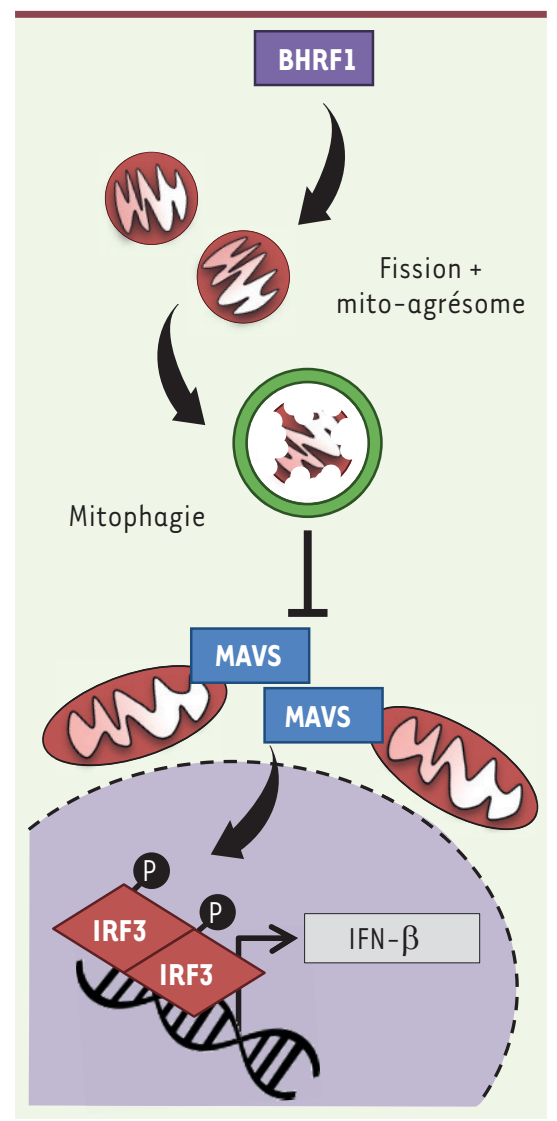

Figure 3. La mitophagie induite par BHRFI est à l'origine de l'inhibition de la réponse interféron. La présence du récepteur MAVS à la surface des mitochondries en fait des organites essentiels dans le déclenchement de l'immunité innée. En induisant la mitophagie, BHRFl réduit cette défense antivirale car la séquestration des mitochondries ainsi que leur dégradation limitent l'activité de MAVS, qui n'est alors plus en mesure d'activer le facteur de transcription IRF3 (interferon regulatory factor 3). Ce dernier ne parvient donc plus au noyau cellulaire, et ne stimule plus la transcription du gène codant IFN- $\beta$.

\section{RÉFÉRENCES}

1. Levine $B$, Sinha $S$, Kroemer $\mathrm{G}$. Bcl-2 family members. Autophagy 2008; 4 : 600-6.

2. Henderson S, Huen D, Rowe M, et al. Epstein-Barr virus-coded BHRFl protein, a viral homologue of $\mathrm{Bcl}-2$, protects human $\mathrm{B}$ cells from programmed cell death. Proc Natl Acad Sci USA 1993 ; $90: 8479-83$

3. Vilmen G, Glon D, Siracusano G, et al. BHRF1, a BCL2 viral homolog, disturbs mitochondrial dynamics and stimulates mitophagy to dampen type I IFN induction. Autophagy 2020 ; may 13 : 1-20. 


\section{RÉFÉRENCES}

4. Liesa M, Palacín M, Zorzano A. Mitochondrial dynamics in mammalian health and disease. Physiol Rev 2009; $89: 799-845$

5. Arnoult D, Soares F, Tattoli I, et al. Mitochondria in innate immunity. EMBO Rep 2011 ; 12 : 901-10.

6. Chang $C R$, Blackstone $C$. Dynamic regulation of mitochondrial fission through modification of the dynamin-related protein Drpl. Ann N Y Acad Sci 2010 ; $1201: 34-9$.

7. Herzig S, Shaw RJ. AMPK: guardian of metabolism and mitochondrial homeostasis. Nat Rev Mol Cell Biol 2018 ; 19 : 121-35.
8. Green DR, Levine B. To be or not to be? How selective autophagy and cell death govern cell fate. Cell 2014 ; $157: 65-75$.

9. Burman J, Pickles S, Wang C, et al. Mitochondrial fission facilitates the selective mitophagy of protein aggregates. J Cell Biol 2017 ; $216: 3231-47$.

10. Randow F, Youle RJ. Self and non-self: How autophagy targets mitochondria and bacteria. Cell Host Microbe 2014 ; 15 : 403-11.

11. Vazquez C, Horner SM. MAVS Coordination of antiviral innate immunity. J Virol $2015 ; 89$ : 6974-7.
12. Watanabe A, Maruo S, Ito T, et al. Epstein-Barr virusencoded $\mathrm{Bcl}-2$ homologue functions as a survival factor in Wp-restricted Burkitt lymphoma cell line P3HR-1.J Virol $2010 ; 84$ : 2893-901.

13. Bekisz J, Baron S, Balinsky C, et al. Antiproliferative properties of type I and type II interferon. Pharmaceuticals (Basel) $2010 ; 3$ : 994-1015.

14. Kim S-J, Syed GH, Siddiqui A. Hepatitis C virus induces the mitochondrial translocation of Parkin and subsequent mitophagy. PLoS Pathog 2013; 9:e1003285.

15. Wang R, Zhu Y, Ren C, et al. Influenza A virus protein $\mathrm{PB1}-\mathrm{F} 2$ impairs innate immunity by inducing mitophagy. Autophagy 2020 ; Feb 11 : 1-16. 